\title{
Custo e caracterização de infecção hospitalar em idosos
}

\author{
Cost and characterization of hospital infection among the elderly
}

Érika Maria Izaias ${ }^{1}$

Mara Solange Gomes Dellaroza ${ }^{1}$

Mariana Ângela Rossaneis ${ }^{1}$

Renata Aparecida Belei ${ }^{1}$

${ }^{1}$ Programa de Gerência dos Serviços de Enfermagem, Departamento de Enfermagem, Centro de Ciências da Saúde, Universidade Estadual de Londrina. Av. Robert Koch 60, Vila Operária. 86.038440 Londrina PR Brasil. erikamizaias@yahoo.com.br
Abstract The increase in the number of elderly individuals has a direct impact on the health system with hospitalization complications such as hospital infection (HI). The objective of this study is to characterize and evaluate the cost of HI among the elderly in a public hospital for the elderly. Descriptive, cross-sectional research was conducted. Elderly individuals with HI in 2010 comprised the population of the study. The data were drawn from hospital records and analysis was performed using Excel 2003 and SPSS software. The results indicated that 341 seniors participated in the study with a HI index 3\% higher than the monthly average. The most frequent infectious sites were: respiratory tract infection (58.1\%), urinary tract infection (28.6\%) and surgical infection (4.6\%). Each hospitalization cost $R \$ 28,714.10$ on average and $5 \%$ of this amount was for antibiotics. The increase of 15 days average added $R \$ 17,946.3$ to costs of hospitalization. The conclusion drawn is that the elderly have increased vulnerability to HI. The infections extend the hospitalization period and increase costs varying according to the nature of the infection.

Key words Hospital infection, Elderly individuals, Healthcare costs, Nursing
Resumo O crescimento de idosos representa impacto direto no setor saúde. A hospitalização predispõe os idosos a complicações como a infecção hospitalar (IH). Objetivo: caracterizar perfil e custos de IH em idosos em um hospital terciário público. Método: estudo analítico, transversal. Idosos com IH em 2010 compuseram a população de estudo. Os dados derivaram de setores intra-hospitalares $e$ as análises dos programas Excel 2010 e SPSS. Resultados: Participaram do estudo 341 idosos com indice de IH 3\% superior à média mensal. Os sítios infecciosos mais frequentes foram: trato respiratório (58,1\%), urinário $(28,6 \%)$ e sítio cirúrgico $(4,6 \%)$. Cada internação custou em média $R \$ 28.714,10$ e a antibioticoterapia representou $5 \%$ desse valor. $O$ aumento de 15 dias à média de permanência acrescentou $R \$ 17.946,30$ aos custos com a internação. Conclusão: o idoso apresenta maior vulnerabilidade à IH. Os quadros infecciosos prolongam internações e elevam custos variando conforme o sitio.

Palavras-chave Infecção hospitalar, Idoso, Custos de cuidados de saúde, Enfermagem 


\section{Introdução}

A população idosa cresce continuamente e representa $10 \%$ entre os brasileiros e a nível mundial deve atingir dois bilhões de pessoas no ano de 2050. A transição do processo demográfico é uma realidade universal devido a mudanças como: diminuição da natalidade, aumento da expectativa de vida, avanço nas pesquisas científicas, acesso aos serviços de saúde e o controle de algumas doenças ${ }^{1-3}$.

A Organização das Nações Unidas (ONU) publica relatórios que estabelecem como idosas as pessoas com 60 anos e mais. Tal critério também é aceito pelas leis brasileiras que regulamentam a assistência a idosos ${ }^{3,4}$.

Os serviços de saúde sofrem impacto direto com o envelhecimento populacional, visto que os idosos apresentam mais problemas de saúde comparados a pessoas mais jovens; pois são propensos à cronicidade de doenças como as do aparelho circulatório e a neoplasias ${ }^{5}$.

A hospitalização é considerada um risco para as pessoas idosas, por serem mais suscetíveis a infecções hospitalares causadas, muitas vezes, pelo repouso prolongado no leito durante o período de internação ${ }^{6}$.

Infecção hospitalar (IH) é toda aquela adquirida durante a internação, normalmente provocada pela microbiota bacteriana humana que, em decorrência da doença, dos procedimentos invasivos e do contato com a microbiota hospitalar, se desequilibra com os mecanismos de defesa do organismo $^{7}$.

Um estudo brasileiro avaliou 332 pacientes em um hospital universitário, dentre os quais $61(18,6 \%)$ apresentaram infecção hospitalar. A taxa geral de mortalidade dos pacientes avaliados foi de $9,8 \%$; já a dos pacientes que tiveram óbito relacionado à infecção hospitalar foi de $22,3 \%{ }^{6}$. Dessa forma, fica evidente que a população acometida com infecção hospitalar apresenta mortalidade maior que aquela não acometida.

Entre os fatores de risco para infecção hospitalar em idosos destacam-se: a presença de comorbidades; neoplasias; neutropenia; a utilização prévia de antimicrobianos; a estadia em unidade de terapia intensiva; a entubação traqueal por mais de 24horas; a internação prolongada ou a transferência para outro hospital ${ }^{8}$. Infecções do trato urinário, pneumonias, infecções do sítio cirúrgico e sepsis são as principais manifestações de infecção hospitalar em idosos?.

Realizou-se um estudo em pacientes com infecção hospitalar na Unidade de Terapia Inten- siva do Hospital São Paulo, em 2002, no qual os autores levantaram dados significantes quanto aos dias de internação de pacientes infectados e não infectados e seus custos; houve um acréscimo de 26 dias de permanência na unidade devido às infecções e isso elevou o custo em valor superior a US\$ 11 mil por paciente ${ }^{10}$.

Os custos com infecções hospitalares subdividem-se em diretos, indiretos ou preventivos e imensuráveis ou intangíveis. Os primeiros representam as despesas com o diagnóstico, que envolve realização de exames e com o tratamento do paciente infectado que abrange medicamentos, diárias adicionais, medidas para precauções e outros exames ${ }^{10}$.

Os custos indiretos ou preventivos correspondem aos investimentos a fim de evitar, reduzir e controlar as infecções hospitalares. Dessa forma, demandam a implantação e a manutenção de um serviço específico como a Comissão de Controle de Infecção Hospitalar (CCIH), onde estão implícitos gastos com área física e salário de profissionais capacitados para desenvolver tais ações ${ }^{10}$.

Há ainda que se ressaltar os custos imensuráveis ou intangíveis, que são a dor e o sofrimento do paciente e de sua família, que afetam a qualidade de vida; pois podem originar a inatividade de algum membro, a perda funcional de um órgão ou, até mesmo, a morte relacionada à infecção hospitalar ${ }^{10}$.

Para reeducar o processo de trabalho assistencial e enfatizar a prática de medidas preventivas, as equipes dos serviços de saúde necessitam conhecer sobre a ocorrência de infecções hospitalares e suas repercussões, tanto para o cliente como para a instituição; com destaque aqui para a assistência às pessoas idosas, grande contingente, hoje, nos serviços.

O presente estudo objetivou caracterizar o perfil e os custos das infecções hospitalares em idosos em um hospital terciário público.

\section{Método}

Trata-se de uma pesquisa do tipo quantitativa, descritiva, transversal e retrospectiva. O estudo foi desenvolvido em um hospital universitário público, que conta com 316 leitos, sete salas cirúrgicas; além de 120 salas de consultas, todos disponíveis para o Sistema Único de Saúde (SUS $)^{11}$.

A população de estudo foi composta por pacientes com 60 anos e mais, notificados com infecção hospitalar no ano de 2010. Os nomes dos 
pacientes não foram utilizados ou divulgados em nenhuma fase da execução da pesquisa.

Os dados coletados foram secundários e provenientes das fichas de notificação da Comissão de Controle de Infecção Hospitalar (CCIH) do hospital. Dados quanto ao número de pacientes internados, o percentual de idosos e a média de permanência destes no ano de 2010, foram obtidos no Serviço de Arquivo Médico e Estatística (SAME) da instituição. Os custos com as doses dos antibióticos foram fornecidos pela Farmácia do hospital, e o setor de Custos disponibilizou dados sobre os custos médios diários por paciente, nas diversas unidades de internação no ano de 2010. Dessa forma, foi possível calcular a média do custo diário da internação dos pacientes idosos que tiveram infecção hospitalar, considerando o período de permanência encontrado durante as coletas.

Os custos médios diários por paciente da instituição incluem os custos diretos e indiretos; sendo os primeiros explícitos e de fácil identificação, compreendidos pelas provisões de recursos humanos e encargos assistenciais diretos, como medicamentos. Já os custos indiretos, giram em torno de questões assistenciais e estão dispersos por diversos departamentos, por exemplo, no aporte tecnológico, portaria, vigilância, central de cópias, diretorias, lavanderias, manutenção predial e higiene hospitalar ${ }^{12,13}$.

As variáveis analisadas foram: faixa etária, sexo, diagnóstico inicial, procedimentos terapêuticos, unidade de internação, dias de internação, sítios infecciosos e antibioticoterapia. Sendo tais variáveis coletadas e armazenadas em banco de dados estruturado no programa Microsoft Excel 2010. A análise estatística ocorreu por meio dos programas Microsoft Excel 2010 e Statistical Package for Social Science (SPSS), sendo realizadas análises descritivas e medidas de dispersão e de tendência central e comparação das médias de custo por sítio infeccioso.

As coletas ocorreram no período de novembro a dezembro de 2011, somente após aprovação do Comitê de Ética e da Diretoria do referido hospital.

\section{Resultados}

Os resultados serão apresentados em quatro partes: caracterização dos idosos internados; caracterização dos idosos com infecção hospitalar; identificação da incidência de infecções hospitalares; e, caracterização do quadro infeccioso.
No período compreendido, entre janeiro e dezembro de 2010, o hospital apresentou um total de 11.177 internações de pacientes de todas as faixas etárias.

\section{Caracterização dos idosos internados}

No período da pesquisa, houve 1998 idosos internados, totalizando 2541 internações, o que representa $22,7 \%$ das internações gerais ocorridas no período. A média mensal de internações de idosos foi de 211,8. A distribuição dos idosos internados quanto ao sexo foi $52 \%$ homens e $48 \%$ mulheres.

\section{Caracterização dos idosos com infecção hospitalar e internação}

Houve 341 idosos com infecção hospitalar (IH) o que representa uma incidência de $17 \%$ de infecção entre aqueles internados no ano de 2010. Durante as análises das fichas de notificação de infecções hospitalares em idosos, foram avaliadas 368, porém 27 foram excluídas por não conterem informações necessárias às análises ou por se apresentarem de forma ilegível, limitando a interpretação das mesmas. Portanto, as perdas foram de $7 \%$ e as fichas incluídas na pesquisa totalizaram 341 documentos. Dos 341 documentos analisados, subdividiu-se o grupo etário entre jovens idosos (60 - 74 anos) que representaram 52\%; idosos (75 -84 anos) correspondendo a $31 \%$ e muito idosos (> 85 anos) com 17\%. A idade máxima encontrada foi de 99 anos e a mínima de 60 anos, a média de idade foi 75 anos, com desvio padrão de 9 anos e mediana de 74 anos. Dentre os 341 idosos houve $55 \%$ de homens e $45 \%$ de mulheres.

Dos 17 diagnósticos iniciais identificados, destacaram-se cinco: neurológicos, cardiovasculares, infecções/sepses, gastrintestinais e ortopédicos com percentuais respectivos de $19,9 \% ; 12,6 \%$; $11,7 \% ; 11,1 \%$ e $10,6 \%$. Para este cálculo foi utilizado somente o primeiro diagnóstico registrado nas fichas de notificação, considerando que este representa o principal dos idosos no momento de internação. Dos 341 idosos avaliados, seis por cento $(6 \%)$ apresentaram mais de um diagnóstico inicial.

Em relação ao tempo de internação, obtevese uma média de 24 dias de permanência para os pacientes idosos que apresentaram infecção hospitalar, com desvio padrão de 18 dias. O tempo mínimo de internação foi de 1 dia e o máximo de 128 dias. Tal média de permanência de internação teve significativa diferença com relação à de idosos sem detecção de infecção hospitalar (9 dias); 
resultando em um acréscimo de 15 dias à média obtida entre os dois grupos.

Foram encontradas 9 unidades de internação nas quais os idosos estiveram internados; contudo, $1 \%$ deles frequentou até 5 diferentes setores da instituição. As principais unidades foram Pronto Socorro, Unidade de Tratamento Intensivo (UTI), Unidade de internação Masculina e Feminina.

Para o cálculo médio dos custos referentes à internação, independente da idade do paciente, levou-se em conta as informações fornecidas pelo Setor de Custos (Tabela 1), relativas à média de custos diretos e indiretos avaliados trimestralmente em cada unidade de internação no ano de 2010.

Entre os idosos com infecção houve um custo médio por paciente de $\mathrm{R} \$ 28.714,08$ com a internação, considerando-se todas as unidades frequentadas e os dias que permaneceram nas mesmas. Levando-se em conta a média de 9 dias de permanência de internação dos idosos sem IH,e o aumento médio de 15 dias de permanência para os que apresentaram $\mathrm{IH}$, obtemos um acréscimo de $\mathrm{R} \$ 17.946,3$ aos custos com a internação.

\section{Identificação da incidência das infecções hospitalares}

No período estudado, houve uma incidência média mensal de $10,1 \%$ de infecções hospitalares entre todos os pacientes internados. Já a incidência de infecções entre idosos foi de 13,4\%.

A incidência mensal das infecções avaliadas no período está exposta no Gráfico 1, considerando as mensais encontradas em nossa pesquisa, bem como as internações de pacientes com 60 anos e mais nos respectivos meses.

Tabela 1. Custo diário médio da internação de pacientes de todas as faixas etárias, de acordo com os setores de internação e trimestres. Londrina - 2010 .

\begin{tabular}{lcccr}
\hline \multicolumn{1}{c}{ Setor } & \multicolumn{3}{c}{$\mathbf{R} \mathbf{}$} \\
\cline { 2 - 5 } & $\mathbf{1}^{\mathbf{0}}$ trimestre & $\mathbf{2}^{\mathbf{0}}$ trimestre & $\mathbf{3}^{\mathbf{0}}$ trimestre & $\mathbf{4}^{\mathbf{0}}$ trimestre \\
\hline UTI & $2.082,23$ & $2.192,43$ & $2.359,83$ & $2.496,87$ \\
Unid. Masculina & 661,16 & 746,53 & 786,82 & 859,43 \\
Unid. Feminina & 731,22 & 870,84 & 862,09 & 947,08 \\
Unid. Moléstias Infecciosas & 685,05 & 743,17 & 804,43 & 778,79 \\
Pronto-socorro & 650,21 & 699,93 & 736,96 & 824,97 \\
Tisiologia & 596,71 & 557,43 & 703,62 & $1.013,58$ \\
Unidade de Queimados & $1.606,93$ & $1.545,19$ & $1.651,23$ & $1.662,66$ \\
\hline
\end{tabular}

* Os valores em reais são referentes aos custos unitários médios calculados a partir do custo total que resulta da soma dos custos diretos e custos indiretos. Fonte: Divisão de finanças e orçamento - Seção de custo hospitalar.

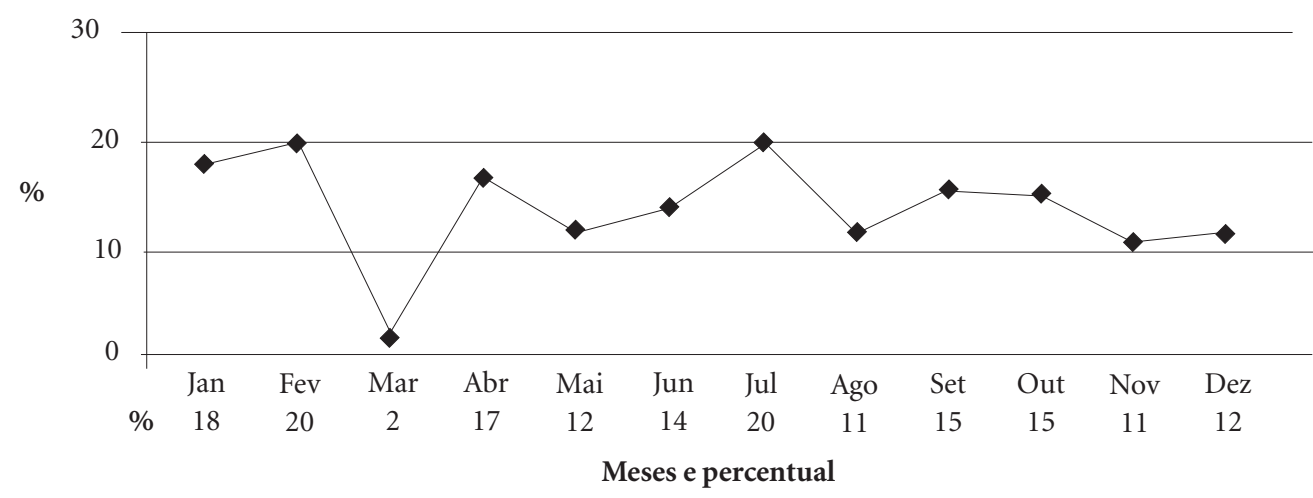

Gráfico 1. Incidência mensal de infecções hospitalares em idosos internados em um hospital universitário público. Londrina- 2010. 


\section{Caracterização do quadro infeccioso}

Foi encontrado um total de 437 registros de infecções hospitalares, sendo que $21,7 \%$ dos idosos apresentaram 2 sítios; em 5,6\% dos idosos foi detectado até 3 e 1,2\% tiveram até 4 tipos.

Durante a coleta de dados, identificou-se 16 tipos de sítios infecciosos; sendo mais frequentes as IH de trato respiratório $(58,1 \%)$, urinário $(28,6 \%)$ e infecção de sítio cirúrgico $(4,6 \%)$. Os demais resultaram em $8,7 \%$ do total.

Em média, o primeiro sítio infeccioso foi detectado 8 dias após a internação. Enquanto que a detecção do segundo sítio de infecção se deu numa média de 19 dias após o início da internação. Para os idosos que apresentaram até 3 e 4 sítios infecciosos, a detecção ocorreu, respectivamente, em uma média de 30 e 43 dias após a entrada no serviço hospitalar.

Após o término de análises e cálculos dos antibióticos, obtivemos um custo médio de R\$ 1.336,90; com desvio padrão de $\mathrm{R} \$ 2.422,80$; custo mínimo de $\mathrm{R} \$ 0,24$ e máximo de $\mathrm{R} \$ 14.866,26$ para a antibioticoterapia empreendida durante a internação dos idosos com IH. Os custos com os antibióticos representaram cerca de 5\% sobre os custos com a internação.

Quanto ao período do tratamento com os antibióticos, verificou-se que houve uma média de 7,5 dias de uso, com desvio padrão de 5,6 dias. O tempo mínimo de uso foi de 1 dia e o máximo de 36. Os custos com as principais infecções estão detalhados na Tabela 2.

Como a variável custos não apresenta padrão normal utilizou-se o teste não paramétrico de Kruskal-Wallis, com ( $\mathrm{p}=0.0001)$, e, através do método de Dunn, verificou-se as diferenças entre os custos dos sítios de infecções mais frequentes. Os resultados demonstraram diferença estatisticamente significante somente na comparação de custos entre as infecções respiratórias e as urinárias, sendo os das primeiras maiores. Todas as outras comparações realizadas não demonstraram diferenças significantes.

\section{Discussão}

A população idosa enfrenta riscos quando se submete às internações hospitalares, pois a permanência no serviço de saúde, o repouso prolongado no leito, a exposição contínua a procedimentos invasivos e os demais fatores que influenciam negativamente a capacidade funcional, precedem alterações da qualidade de vida (Creditor apud Siqueira et al. $)^{5}$. Pacientes idosos apresentam taxas elevadas de internação hospitalar e maior média de permanência na instituição de saúde, quando comparada à população de menos idade ${ }^{5}$.

A pesquisa descreveu como a ocorrência de infecção hospitalar em pacientes idosos gera repercussões diretas e indiretas, incluindo custos elevados para o serviço, maior tempo de permanência nas unidades de internação; o que eleva os riscos quanto às infecções secundárias; Haley (apud Bôas) observou que "a ocorrência de infecção hospitalar determina um aumento no tempo de internação (de quatro dias em média) e dos custos de internação"'.

A média de permanência de internação dos pacientes que apresentaram infecção hospitalar resultou em um acréscimo de 15 dias em relação à média dos idosos que não foram detectados com IH no período, o que remete a aumento dos custos com a internação, levando-se em conta o tratamento com antibióticos, exames e demais procedimentos.

O estudo apontou que a incidência média mensal de infecções hospitalares entre os idosos foi cerca de 3\% maior que aquela de IH detectadas nos pacientes não idosos, no mesmo período e local de estudo. Tal fato indica a vulnerabilidade dos idosos em contrair IH em relação a pacientes jovens e adultos assistidos. Isto se deve ao fato de

Tabela 2. Comparação dos custos de infecção conforme os sítios mais frequentes apresentados pelos idosos internados em um hospital universitário público, Londrina -2010.

\begin{tabular}{lrcrrr}
\hline \multicolumn{1}{c}{ Sítio de infecção } & Média $^{*}$ & Desvio padrão $^{*}$ & \multicolumn{1}{c}{ IC 95\% } & Mediana $^{*}$ & Variância $^{*}$ \\
\hline Trato respiratório & $1.629,80$ & $2.772,30$ & $1.287,20-1.972,40$ & 348,90 & $7.685 .435,9$ \\
Trato urinário & 915,50 & $1.792,30$ & $598,20-1.232,80$ & 149,60 & $3.212 .250,4$ \\
Sítio cirúrgico & $1.032,10$ & $2.218,80$ & $6,30-2.070,50$ & 67,50 & $4.922 .956,9$ \\
Outros & 926 & $1.382,70$ & $471,50-1.380,50$ & 165 & 1.911 .826 \\
\hline
\end{tabular}

* Os valores apresentados referem-se ao custo hospitalar em reais conforme sítio de infecção. 
apresentarem com maior frequência patologias crônico-degenerativas, alterações imunológicas, deficiência da circulação sanguínea e cicatrização tecidual prejudicada. A senescência, em si, contribui diretamente para o desenvolvimento de alterações patológicas ${ }^{7,14}$.

A partir dos anos 1970, a Organização Mundial da Saúde (OMS) organizou um aprofundamento dos estudos a fim de qualificar as infecções hospitalares em diversos países. Entretanto, concluiu-se que a incidência de IH é extremamente variável entre as instituições de saúde e as diferentes regiões; portanto, não há índice aceitável de infecção hospitalar, de forma que o esperado é sua não ocorrência. Na pesquisa, constatou-se uma taxa média mensal de $13,4 \%$ de IH entre idosos ${ }^{7,15}$.

O Centro para Controle de Doenças (Centers for Diseases Control-CDC) de Atlanta realizou estudos, nos EUA, que mostraram que complicações infecciosas no ambiente hospitalar resultam em aumento médio de 4 dias ao tempo de internação, com acréscimo de US $\$ 1.800,00$ aos custos assistenciais ${ }^{7}$. Segundo MMWReport, apud Couto et al., 20097:

O CDC estimou valores entre US\$680, para os custos adicionais com infecção hospitalar do trato urinário, e US\$5.683, para cada episódio de pneumonia, sendo o custo médio com qualquer episódio de infecção hospitalar em torno de US\$2.100.

No Brasil, a Lei 9.431 de 6 de janeiro de 1997 obriga os hospitais a possuírem um programa de controle de complicações infecciosas hospitalares. A criação da Comissão de Controle de Infecção Hospitalar (CCIH) nas instituições hospitalares foi determinada pela Portaria 2.616/GM de 12 de maio de 1998. Tal serviço deve ser composto por representantes das áreas de enfermagem, medicina, farmácia, microbiologia e administração, visando controlar as complicações infecciosas por meio de vigilância epidemiológica, a fim de investigar surtos de infecções, implementar intervenções, bem como avaliar a eficácia das mesmas ${ }^{7}$.

A educação da equipe, a consideração dos princípios de técnica cirúrgica e os cuidados perioperatórios, a desinfecção e o manuseio correto dos equipamentos; as precauções padrão de higienização das mãos, o uso de luvas e aventais; a prevenção de broncoaspiração; o posicionamento adequado de cateteres e sacos coletores de diurese, bem como a observação da necessidade de troca dos mesmos, são algumas medidas preventivas de infecções hospitalares facilmente empregadas e menos onerosas.

Tais medidas devem fazer parte da rotina de trabalho da equipe assistencial, que ao compreen- der a importância das mesmas as associará às suas responsabilidades profissionais ${ }^{7}$.

Pensando na qualidade de vida da pessoa idosa que precisa de atenção à saúde, no seu contentamento e de sua família, o Serviço de Assistência Domiciliar (SAD) pode ser alternativa segura de serviço de saúde. Tal modo de assistência se trata de atenção domiciliar, visando promoção e prevenção da saúde; se caracteriza por atendimento domiciliar quando as atividades desenvolvidas são de cunho ambulatorial e de maneira contínua; e pode ser ainda internação domiciliar, quando se trata atenção em tempo integral para usuários com maior complexidade no quadro clínico, fazendo-se necessário o uso de medicamentos, equipamentos e transporte. Dessa forma, há maior possibilidade de diminuição dos custos assistenciais, preservação da individualidade do idoso, mantendo seus vínculos familiares e prevenção das situações que possam acentuar a perda das capacidades funcionais; evitando as internações que causam inúmeros riscos ${ }^{16-18}$.

Possuir saúde na fase idosa não se resume à falta de doença, mas inclui também a preservação da capacidade funcional, a qual abrange habilidades físicas e mentais essenciais para que se tenha independência e autonomia. A partir da avaliação funcional do idoso haverá a possibilidade de planejar uma assistência direcionada à prevenção, pois os diversos graus de incapacidade contribuirão diretamente para o aumento de agravos à saúde, bem como para aumento do risco de quedas e, consequentemente, utilização dos serviços de saúde e submissão aos riscos dos hospitais ${ }^{16,17}$.

A pesquisa encontrou limitação pelo fato de utilizar dados secundários que apresentavam registros, por vezes, incompletos; dificultando principalmente a identificação de microrganismos e procedimentos associados aos processos infecciosos.

\section{Conclusões e Considerações Finais}

As infecções hospitalares remetem a um antigo problema das instituições assistenciais e, desde que ganharam espaço entre as discussões das organizações de saúde, geram inúmeros estudos que consideram a importância de sua prevenção, suas principais causas e suas repercussões, entre elas os custos diretos e indiretos, ao serviço e aos pacientes.

Uma vez adoecidos e com necessidade de internação, os pacientes são expostos aos riscos do ambiente hospitalar. Contudo, tais riscos se 
acentuam para alguns grupos, sendo um deles composto pelos idosos, que devido ao processo de imunossenescência, às doenças crônico-degenerativas e à toda fisiologia do envelhecimento, se encontram mais suscetíveis às complicações infecciosas que acabam por estender o tempo de convalescença.

Cursos relacionados às ciências da saúde têm a necessidade de conhecer os serviços de prevenção e controle de infecção hospitalar, por meio da inclusão de tais conteúdos nos currículos disciplinares, visto que trata-se de um tema imprescindível a todos os envolvidos nos processos assistenciais; aumentando assim a percepção do custo/benefício de medidas preventivas.

Dentre as questões preventivas encontramos também o sistema de assistência domiciliar como tema emergente quanto à qualidade de vida dos idosos.

No presente estudo, a infecção hospitalar triplicou o tempo médio de permanência no serviço e os idosos foram detectados com complicação infecciosa cerca de uma semana após serem admitidos na instituição; quase $1 / 4$ (um quarto) dos idosos apresentou mais de um tipo de infecção. Tais fatos apontam para exposição demasiada aos riscos hospitalares; possíveis readmissões e consequente elevação de custos.

Levando em consideração os serviços de referência e contrarreferência envolvidos no setor saúde, observa-se a necessidade de se explorar o conhecimento quanto às medidas de prevenção; inicialmente à saúde do idoso na comunidade visando preservar sua capacidade funcional diante do processo e envelhecimento. Posteriormente, mantendo vigilância epidemiológica dentro dos serviços hospitalares que recebem esse idoso, a fim de diagnosticar as fragilidades assistenciais que contribuem para as complicações infecciosas, realizar a implantação de medidas direcionadas à prevenção e ao controle e avaliar a efetividade das mesmas para traçar, então, metas subsequentes.

\section{Colaboradores}

EM Izaias, MSG Dellaroza, MA Rossaneis e RA Belei participaram igualmente de todas as etapas de elaboração do artigo. 


\section{Referências}

1. Loyola Filho A, Matos DL, Giatti L, Afradique ME, Peixoto SV, Lima-Costa MF. Causas de internações hospitalares entre idosos brasileiros no âmbito do Sistema Único de Saúde. Epidemiologia e serviços de saúde 2004; 13(4):229-238.

2. Instituto Brasileiro de Geografia e Estatística (IBGE). Primeiros resultados definitivos do Censo 2010. [acessado 2012 jul 20]. Disponível em: http://www.ibge. gov.br/home/presidencia/noticias/noticia_visualiza. php?id_noticia $=18668$ id_pagina $=1$

3. Brasil. Organização das Nações Unidas (ONU). A ONU e as pessoas idosas. [acessado 2012 set 21]. Disponível em: http://www.onu.org.br/a-onu-em-acao/a-onu-em -acao/a-onu-e-as-pessoas-idosas/

4. Brasil. Presidência da República. Lei 10.741 , de $1^{\circ}$ de outubro de 2003. Dispõe sobre o Estatuto do Idoso e dá outras providências. Diário Oficial da União 2003; 3 out.

5. Siqueira AB, Cordeiro RC, Perracini MR, Ramos RL. Impacto funcional da internação hospitalar de pacientes idosos. Rev Saude Publica 2004; 38(5):687-694.

6. Bôas PFV, Ruiz T. Ocorrência de infecção hospitalar em idosos internados em um hospital universitário. Rev Saude Publica 2004; 38(3):372-378.

7. Couto RC, Pedrosa TMG, Cunha AFA, Amaral DB. Infecção hospitalar e outras complicações não-infecciosas da doença: epidemiologia, controle e tratamento. $4^{\text {a }}$ ed. Rio de Janeiro: Guanabara Koogan; 2009.

8. Sax H, Pittet D. Interhospital differences in nosocomial infection rates: importance of case-mix adjustment. Arch Intern Med 2002; 162(21):2437-2442.

9. Fernandes AT, Fernandes MOV, Ribeiro Filho N. Infecção hospitalar e suas interfaces na área da saúde. São Paulo: Atheneu; 2000.

10. Andrade GM. Custos da infecção hospitalar e o impacto na área da saúde. BSBM Brasília Med 2005; 42(1/2):4850.

11. Hospital Universitário Regional do Norte do Paraná. Universidade Estadual de Londrina. 2011. [acessado 2011 maio 25]. Disponível em: http://www.hu.uel.br/ index.php?pagina $=29 \&$ pai $=7$
12. Kurcgant P. Gerenciamento em enfermagem. Rio de Janeiro: Guanabara Koogan; 2005.

13. Marquis BL, Huston CJ. Administração e liderança em enfermagem: teoria e prática. $4^{\mathrm{a}}$ ed. Porto Alegre: Artmed; 2005. p. 112.

14. Oliveira DR, Lenardt MH, Tuofo FS. O idoso e o sistema de cuidado à saúde na doença renal. Acta Paul Enf 2003; 16(4):49-58

15. Brasil. Agência Nacional de Vigilância Sanitária (Anvisa). Sistema de Perguntas e Respostas. 2002. [acessado 2012 set 19]. Disponível em: http://www.anvisa.gov.br/faqdinamica/index.asp?Secao $=$ Usuario\&usersecoes $=30 \& u-$ serassunto $=49$

16. Freitas EV, Py L, Neri AL, Cançado FAX, Gorzoni ML, Rocha SM, organizadores. Tratado de geriatria e gerontologia. $2^{\mathrm{a}}$ ed. Rio de Janeiro: Guanabara Koogan; 2006. p. 1122-1125.

17. Chiossi JSC, Roque FP, Goulart B, Chiari B M. Impacto das mudanças vocais e auditivas na qualidade de vida de idosos ativos. Cienc Saude Colet 2012; 19(8):3335-3342.

18. Araújo LUA, Gama ZAS, Nascimento FLA, Oliveira HFV, Azevedo WM, Almeida Júnior HJB. Avaliação da qualidade de atenção primária à saúde sob a perspectiva do idoso. Cien Saude Colet 2013; 19(8):3521-3532.

Artigo apresentado em 28/07/2013

Aprovado em 29/08/2013

Versão final apresentada em 02/09/2013 